\title{
Next Steps in Improving Healthcare Value: Postacute Care Transitions: Develop- ing a Skilled Nursing Facility Collaborative within an Academic Health System
}

\author{
Sarah J Conway, MD ${ }^{1 *}$; Anand K Parekh, MD, MPH ${ }^{1,2}$; Andrew H Hughes, MD1,3; \\ Carol Sylvester, RN, MS'; Sarah Himmelrich, MPH'; Lindsay C Hebert, MSPH'; Damien Doyle, MD'; \\ Michele Bellantoni, MD'; Scott A Berkowitz, MD, MBA'
}

${ }^{1}$ The Johns Hopkins School of Medicine, Baltimore, Maryland; '2Bipartisan Policy Center, Washington, DC; ${ }^{3}$ Johns Hopkins Bayview Medical Center, Baltimore, Maryland; ${ }^{4}$ Johns Hopkins HealthCare LLC, Glen Burnie, Maryland; On behalf of the Johns Hopkins Medicine Skilled Nursing Facility Collaborative

Hospitals are under financial pressure to shorten hospitalizations and reduce readmissions. Current evidence suggests that postacute care-associated rehospitalizations could be reduced by focusing on a concentrated referral network of preferred high-quality skilled nursing facilities (SNFs). Hospitals, health systems, and health plans have taken several approaches to creating preferred provider networks to streamline and improve the quality of SNF discharges. We propose a collaborative framework for the establishment of a preferred postacute care network based on the experience of the Johns Hopkins Medicine Skilled Nursing Facility Collaborative and review early implementation challenges. Journal of Hospital Medicine 2019;14:174-177. (C) 2019 Society of Hospital Medicine

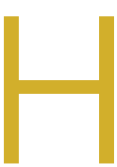

ospitals and health systems are under mounting financial pressure to shorten hospitalizations and reduce readmissions. These priorities have led to an ever-increasing focus on postacute care (PAC), and more specifically on improving transitions from the hospital. ${ }^{1,2}$ According to a 2013 Institute of Medicine report, PAC is the source of $73 \%$ of the variation in Medicare spending ${ }^{3}$ and readmissions during the postacute episode nearly double the average Medicare payment. ${ }^{4}$ Within the PAC landscape, discharges to skilled nursing facilities (SNFs) have received particular focus due to the high rates of readmission and associated care costs. ${ }^{5}$

Hospitals, hospital physicians, PAC providers, and payers need to improve SNF transitions in care. Hospitals are increasingly responsible for patient care beyond their walls through several mechanisms including rehospitalization penalties, value-based reimbursement strategies (eg, bundled payments), and risk-based contracting on the total cost of care through relationships with accountable care organizations (ACOs) and Medicare Advantage plans. Similarly, hospital-employed physicians and PAC providers are more engaged in achieving value-based goals through increased alignment of provider compensation models $s^{6,7}$ with risk-based contracting.

Current evidence suggests that rehospitalizations could be reduced by focusing on a concentrated referral network of preferred high-quality SNFs; 8,9 however, less is known about how to develop and operate such linkages at the administrative or

*Corresponding Author: Sarah Johnson Conway, MD; Telephone: 410-5020668; E-mail: sjc@jhmi.edu

Received: July 24, 2018; Revised: October 24, 2018;

Accepted: October 27, 2018

(C) 2019 Society of Hospital Medicine DOI 10.12788/jhm.3117 clinical levels. ${ }^{8}$ In this article, we propose a collaborative framework for the establishment of a preferred PAC network.

\section{SKILLED NURSING FACILITY PREFERRED PRO- VIDER NETWORK}

One mechanism employed to improve transitions to SNFs and reduce associated readmissions is to create a preferred provider network. Increasing the concentration of hospital discharges to higher performing facilities is associated with lower rehospitalization rates, particularly during the critical days following discharge. ${ }^{10}$

While the criteria applied for preferred provider networks vary, there are several emerging themes. ${ }^{10}$ Quality metrics are often applied, generally starting with Centers for Medicare and Medicaid Services (CMS) quality star ratings and Long-Term Care Minimum Data Set (MDS) metrics with additional criteria frequently layered upon those. Some examples include the extent of physician coverage, ${ }^{11}$ the extent of nursing coverage (eg, nursing ratios or $24 / 7$ nursing care), geographic access, and flexible admission times (including weekends and nights). ${ }^{12}$ In addition, several outcome measures may be used such as 30-day readmission rates, patient/ family satisfaction ratings, ED visits, primary care follow-up within seven days of PAC discharge, or impact on the total cost of care.

Beyond the specified criteria, some hospitals choose to build upon existing relationships when developing their preferred network. By selecting historically high-volume facilities, they are able to leverage the existing name recognition amongst patients and providers. ${ }^{13}$ This minimizes retraining of discharge planners, maintains institutional relationships, and aligns with the patients' geographic preferences. ${ }^{2,13}$ While the high volume SNFs may not have the highest quality ratings, some hospitals find they can leverage the value of preferred partner status to push behavior change and improve performance..$^{13}$ 


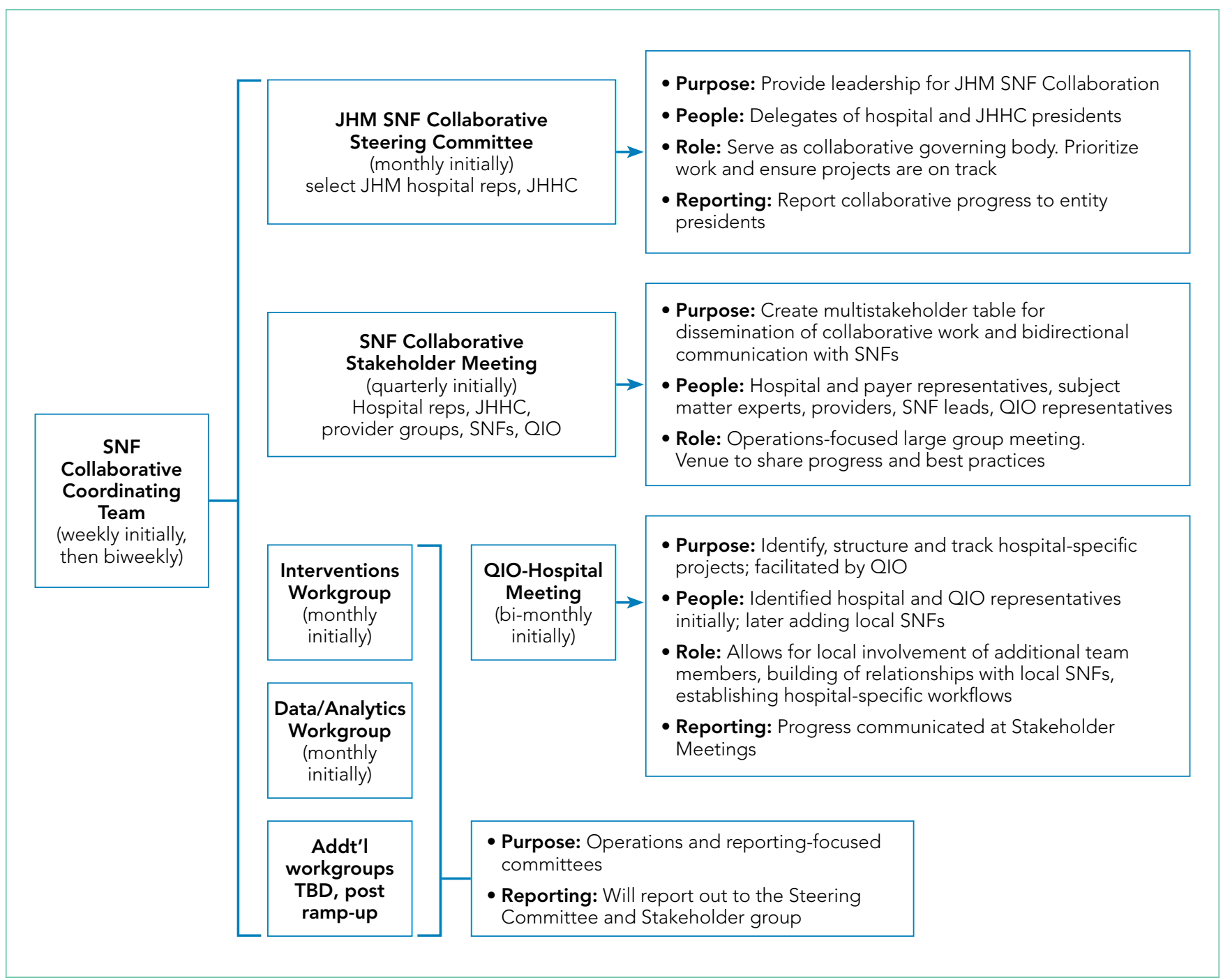

FIG. Skilled Nursing Facility Collaborative Governance Structure.

Abbreviations: JHM, John Hopkins Medicine; JHHC, Johns Hopkins HealthCare; QIO, quality improvement organization; SNF, skilled nursing facility.

\section{PROPOSED HEALTH SYSTEM FRAMEWORK FOR CREATING A SKILLED NURSING FACILITY COLLABORATIVE}

Here we propose a framework for the establishment of a preferred provider network for a hospital or health system based on the early experience of establishing an SNF Collaborative within Johns Hopkins Medicine (JHM). JHM is a large integrated health care system, which includes five hospitals within the region, including two large academic hospitals and three community hospitals serving patients in Maryland and the District of Columbia. ${ }^{14}$

JHM identified a need for improved coordination with PAC providers and saw opportunities to build upon successful individual hospital efforts to create a system-level approach with a PAC partnership sharing the goals of improving care and reducing costs. Additional opportunities exist given the unique Maryland all-payer Global Budget Revenue system managed by the Health Services Cost Review Commission. This system imposes hospital-level penalties for readmissions or poor quality mea- sure performance and is moving to a new phase that will place hospitals directly at risk for the total Part A and Part B Medicare expenditures for a cohort of attributed Medicare patients, inclusive of their PAC expenses. This state-wide program is one example of a shift in payment structures from volume to value that is occurring throughout the healthcare sector.

Developing a formal collaboration inclusive of the five local hospitals, Johns Hopkins HealthCare (JHHC) — the managed care division of JHM - and the JHM ACO (Johns Hopkins Medicine Alliance for Patients, JMAP), we established a JHM SNF Collaborative. This group was tasked with improving the continuum of care for our patients discharged to PAC facilities. Given the number and diversity of entities involved, we sought to draw on efforts already managed and piloted locally, while disseminating best practices and providing added services at the collaborative level. We propose a collaborative multistakeholder model (Figure) that we anticipate will be adaptable to other health systems.

At the outset, we established a Steering Committee and a broad Stakeholder Group (Figure). The Steering Committee is 
TABLE. Initial Intervention Workgroup Priorities

\begin{tabular}{ll}
\hline Priority & Example \\
\hline Upgrading shared EMR transitions documentation & $\begin{array}{l}\text { Recommend changes to the universal discharge summary template. Changes include more logical order } \\
\text { such as prioritizing medications, adding advanced care planning and capacity documentation, incorporating } \\
\text { functional status and standardized assessments, and added recommendations for the next phase of care }\end{array}$ \\
\hline Standardizing metrics for physical, cognitive, and functional status & $\begin{array}{l}\text { Broadly incorporating AM-PAC'M score into rehabilitation therapy assessments and discharge materials for } \\
\text { objective measurement of functional status }\end{array}$ \\
\hline Identifying prediction tools for optimal postdischarge locations & Reviewing landscape of risk-prediction tools for specific patient populations \\
\hline Developing uniform patient/caregiver education materials & $\begin{array}{l}\text { Draft patient education booklet on SNF basics and 'what to expect' to be integrated into the EMR patient } \\
\text { education platform. Planning patient education video on postacute care options }\end{array}$ \\
\hline Outlining strategies to increase provider communication and improve discharge handoffs & $\begin{array}{l}\text { In addition to improving discharge documentation, review strategies to improve the accessibility of hospital } \\
\text { providers after discharge }\end{array}$
\end{tabular}

Abbreviations: AM-PAC, activity measure for postacute care; EMR, electronic medical record.

comprised of representatives from all participating JHM entities and serves as the collaborative governing body. This group initially identified 36 local SNF partners including a mixture of larger corporate chains and freestanding entities. In an effort to respect patient choice and acknowledge geographic preferences and capacity limitations, partner selection was based on a combination of publically available quality metrics, historic referral volumes, and recommendations of each JHM hospital. While we sought to align with high-performing SNFs, we also saw an opportunity to leverage collaboration to drive improvement in lower-performing facilities that continue to receive a high volume of referrals. The Stakeholder Group includes a broader representation from JHM, including subject matter experts from related medical specialties (eg, Physical Medicine and Rehabilitation, Internal Medicine, Emergency Medicine, and various surgical subspecialties); partner SNFs, and the local CMS-funded Quality Improvement Organization (Q|O). Physician leadership was essential at all levels of the collaborative governing structure including the core Coordinating Team (Figure). Providers representing different hospitals were able to speak about variations in practice patterns and to assess the feasibility of suggested solutions on existing workflows.

After establishing the governance framework for the collaborative, it was determined that dedicated workgroups were needed to drive protocol-based initiatives, data, and analytics. For the former, we selected transitions of care as our initial focus area. All affiliated hospitals were working to address care transitions, but there were opportunities to develop a harmonized approach leveraging individual hospital input. The workgroup included representation from medical and administrative hospital leadership, JHHC, JMAP, our home care group, and SNF medical leadership. Initial priorities identified are reviewed in the Table. We anticipate new priorities for the collaborative over time and intend for the workgroup to evolve in line with shifting priorities.

We similarly established a multidisciplinary data and analytics workgroup to identify resources to develop the SNF, and a system-level dashboard to track our ongoing work. While incorporating data from five hospitals with varied patient pop- ulations, we felt that the risk-adjusted PAC data were critical to the collaborative establishment and goal setting. After exploring internal and external resources, we initially elected to engage an outside vendor offering risk-adjusted performance metrics. We have subsequently worked with the state health information exchange, CRISP, 15 to develop a robust dashboard for Medicare fee-for-service beneficiaries that could provide similar data.

\section{IMPLEMENTATION}

In the process of establishing the SNF Collaborative at JHM, there were a number of early challenges faced and lessons learned:

- In a large integrated delivery system, there is a need to balance the benefits of central coordination with the support for ongoing local efforts to promote partner engagement at the hospital and SNF level. The forums created within the collaborative governance structure can facilitate sharing of the prior health system, hospital or SNF initiatives to grow upon successes and avoid prior pitfalls.

- Early identification of risk-adjusted PAC data sources is central to the collaborative establishment and goal setting. This requires assessment of internal analytic resources, budget, and desired timeline for implementation to determine the optimal arrangement. Similarly, identification of available data sources to drive the analytic efforts is essential and should include a health information exchange, claims, and MDS among others.

- Partnering with local QIOs provides support for facility-level quality improvement efforts. They have the staff and onsite expertise to facilitate process implementation within individual SNFs.

- Larger preferred provider networks require considerable administrative support to facilitate communication with the entities, coordinate completion of network agreements, and manage the dissemination of SNF- and hospital-specific performance data.

- Legal and contractual support related to data sharing and HIPAA compliance is needed due to the complexity of the health system and SNF legal structure. Multiple JHM legal 
entities were involved in this collaborative as were a mixture of freestanding SNFs and corporate chains. There was a significant effort required to execute both data-sharing agreements as well as charters to enable QIO participation.

- Physician leadership and insight are key to implementing meaningful and broad change. When devising system-wide solutions, incorporation and respect for local processes and needs are paramount for provider engagement and behavior change. This process will likely identify gaps in understanding the PAC patient's experience and needs. It may also reveal practice variability and foster opportunities for provider education on the needs of PAC teams and how to best facilitate quality transitions.

\section{CONCLUSION}

We proposed a framework for establishing a collaborative partnership with a preferred network of SNF providers. Depending on organizational readiness, significant upfront investment of time and resources could be needed to establish a coordinated net- work of SNF providers. However, once established, such networks can be leveraged to support ongoing process improvement efforts within a hospital or delivery system and can be used strategically by such health systems as they implement value-based health strategies. Furthermore, the lessons learned from transitions to SNFs can be applied more broadly in the PAC landscape including transitions to home from both the hospital and SNF.

\section{Acknowledgments}

The authors wish to acknowledge all the members and participants in the Johns Hopkins Medicine Skilled Nursing Facility Collaborative and the executive sponsors and JHM hospital presidents for their support of this work.

Disclosures: Michele Bellantoni receives intramural salary support for being the medical director of the JHM SNF Collaborative. Damien Doyle is a part-time geriatrician at the Hebrew Home of Greater Washington, a skilled nursing facility. He received travel expense support for GAPNA, a local Advanced Practice Nurse Association meeting. The authors otherwise have no potential conflicts of interest to disclose.

Funding: The authors state that there were no external sponsors for this work.

\section{References}

1. Burke RE, Whitfield EA, Hittle D, et al. Hospital readmission from post-acute care facilities: risk factors, timing, and outcomes. J Am Med Dir Assoc. 2016;17(3):249-255. doi:10.1016/j.jamda.2015.11.005.

2. Mchugh JP, Zinn J, Shield RR, et al. Strategy and risk sharing in hospitalpost-acute care integration. Health Care Manage Rev. 2018:1. doi:10.1097/ hmr.0000000000000204.

3. Institute of Medicine. Variation in Health Care Spending Assessing Geographic Variation.; 2013. http://nationalacademies.org/hmd/ /media/Files/Report Files/2013/Geographic-Variation2/geovariation_rb.pdf. Accessed January 4, 2018.

4. Dobson A, DaVanzo JE, Heath S, et al. Medicare Payment Bundling: Insights from Claims Data and Policy Implications Analyses of Episode-Based Payment. Washington, DC; 2012. http://www.aha.org/content/12/ahaaamcbundlingreport.pdf. Accessed January 4, 2018.

5. Mor , Intrator $\mathrm{O}$, Feng Z, Grabowski DC. The revolving door of rehospitalization from skilled nursing facilities. Health Aff. 2010;29(1):57-64. doi:10.1377/ hlthaff.2009.0629.

6. Torchiana DF, Colton DG, Rao SK, Lenz SK, Meyer GS, Ferris TG. Massachusetts general physicians organization's quality incentive program produces encouraging results. Health Aff. 2013;32(10):1748-1756. doi:10.1377/ hlthaff.2013.0377.

7. Michtalik HJ, Carolan HT, Haut ER, et al. Use of provider-level dashboards and pay-for-performance in venous thromboembolism prophylaxis. J Hosp Med. 2014;10(3):172-178. doi:10.1002/jhm.2303.

8. Rahman M, Foster AD, Grabowski DC, Zinn JS, Mor V. Effect of hospital-SNF

referral linkages on rehospitalization. Health Serv Res. 2013;48(6pt1):18981919. doi:10.1111/1475-6773.12112.

9. Huckfeldt PJ, Weissblum L, Escarce JJ, Karaca-Mandic P, Sood N. Do skilled nursing facilities selected to participate in preferred provider networks have higher quality and lower costs? Health Serv Res. 2018. doi:10.1111/14756773.13027.

10. American Hospital Association. The role of post-acute care in new care delivery models. TrendWatch. http://www.aha.org/research/reports/tw/15dec-twpostacute.pdf. Published 2015. Accessed December 19, 2017.

11. Lage DE, Rusinak D, Carr D, Grabowski DC, Ackerly DC. Creating a network of high-quality skilled nursing facilities: preliminary data on the postacute care quality improvement experiences of an accountable care organization. J Am Geriatr Soc. 2015;63(4):804-808. doi:10.1111/jgs.13351.

12. Ouslander JG, Bonner A, Herndon L, Shutes J. The Interventions to Reduce Acute Care Transfers (INTERACT) quality improvement program: an overview for medical directors and primary care clinicians in long-term care. J Am Med Dir Assoc. 2014;15(3):162-170. doi:10.1016/j.jamda.2013.12.005.

13. McHugh JP, Foster $A$, Mor $V$, et al. Reducing hospital readmissions through preferred networks of skilled nursing facilities. Health Aff. 2017;36(9):15911598. doi:10.1377/hlthaff.2017.0211.

14. Fast Facts: Johns Hopkins Medicine. https://www.hopkinsmedicine.org/ about/downloads/JHM-Fast-Facts.pdf. Accessed October 18, 2018.

15. CRISP - Chesapeake Regional Information System for our Patients. https:// www.crisphealth.org/. Accessed October 17, 2018. 